\title{
EL SENTIDO DEL CONCEPTO. UN ESTUDIO A TRAVÉS DE TOMÁS DE AQUINO
}

\author{
Víctor Velarde-Mayol \\ University of Illinois at Urbana-Champaign
}

El término «concepto» tiene múltiples significados, aquí me limitaré al sentido restringido de lo que posteriormente a Tomás de Aquino se ha llamado «concepto formal». En este sentido, el concepto es algo producido y dicho cuando la operación intelectual llega a término en el logro de una verdad. El presente estudio se ceñirá a dos breves cuestiones clásicas: $\left.1^{\mathfrak{a}}\right)$ en qué sentido el concepto es lo preconocido, y $2^{\mathfrak{a}}$ ) en qué sentido el concepto es alteridad, por el que se conoce al otro en calidad de otro.

\section{EL CONCEPTO COMO «PRECONOCIDO»}

El propio Tomás de Aquino afirma claramente que lo primero entendido es el concepto y lo segundo entendido es la cosa que representa, ${ }^{1}$ ya que no parece posible que el concepto haga conocer lo que representa sin hacerse conocer. Además, una semejanza no puede cumplir la función de imagen si antes no es conocida, en otras palabras, lo que convierte una semejanza en imagen es precisamente su previo conocimiento, no cabe una imagen desconocida, si en cambio una semejanza. ${ }^{2}$ La semejanza implica únicamente una relación real con el asemejado, mientras la imagen añade una relación cognoscitiva, que es lo que se exige para la índole de

1 «Primum intellectum est similitudo rei intellectae, in intellectu existens; et secundum intellectum est ipsa res quae per similitudinem intelligitur». I Sent., d.35, q.1, a.2. El objeto entendido ha de estar en el que entiende en su ser intencional y no real o físico. Como el concepto es necesario para la intelección de objetos ausentes, entonces, «lo entendido, en cuanto está en el que entiende, es una cierto verbo del entendimiento». Compendium Theologiae, I, c.37, n.71.

2 «Similitudo enim significat relationem causatam ex unitate qualitatis, quae relatio requirit distincta supposita, est enim similitudo rerum differentium eadem qualitas». I Sent., d.2, q.1 exp. La semenzan implica esencialmente una relación real pero no necesariamente cognoscitiva, que es intencional, no-real. 
objeto qua objeto. De aquí se desprende, que el concepto se lo ha de conocer primero al menos en el orden de la naturaleza. Ciertamente, para que se pueda dar esta relación cognoscitiva se exige fundamentalmente en la imagen una relación al origen, algo del cual proceda la semejanza, que es una relación real. Es esto en realidad lo que hace que la intención se dirija al objeto. ${ }^{3}$ El reconocimiento de la relación de la imagen a su origen es lo que hace que una semejanza sea imagen. Aquí me interesa más resaltar el aspecto cognoscitivo de la imagen, que utilizaré en lo sucesivo.

El acto del intelecto y el concepto se constituyen en una unidad en relación a lo conocido, en donde el concepto es una semejanza perfecta, generada y expresada por el propio intelecto. El concepto es todo lo expresado por el intelecto y toda la expresión de la cosa dicha, pero también es todo en el que la cosa se expresa. Esto explica en parte que el concepto es lo entendido principalmente, puesto que la cosa no se la entiende sino en el concepto. ${ }^{4}$ De aquí se desprende que el concepto es lo que (quod) se entiende. En este punto el ejemplo del espejo es bastante ilustrativo. Lo que primeramente se ve en el orden de la naturaleza es la propia imagen reflejada en el espejo, y mediante ésta la cosa representada.

No obstante, el intelecto no juzga acerca del concepto como semejanza, esto es ya función del juicio como operación que compone y divide. El intelecto concibe de un modo simple (incomplejo) la semejanza del hombre como la de un animal racional y mortal, pero no sabe que la tiene como semejanza, ya que no juzga que el hombre es animal racional y mortal. Es en el juicio donde se tiene una reflexión sobre el concepto conociéndolo como semejanza de la cosa. ${ }^{5}$ El conocimiento por adecuación veritativa es precisamente esto, que puede quedar expresado de la siguiente manera: conocer el concepto como semejanza no es sólo un conocimiento de sí mismo, sino también de la cosa como término de la adecuación. ${ }^{6} \mathrm{El}$ juicio conoce la adecuación veritativa, y esto exige conocer dos términos (la esencia de la adecuación

3 «De ratione imaginis est similitudo. Non autem quaecumque similitudo sufficit ad rationem imaginis (...) sed requiritur ad rationem imaginis origo. (...) Ad hoc ergo, quod vere aliquid sit imago, requiritur, quod ex
alio procedat simile ei in specie vel saltem in signo speciei». Summa Th., I, q.35, a.1.

4 «Sicut in principio actionis, intellectus et species non sunt duo, sed unum est ipse intellectus et species illustratae; ita unum in fine relinquitur, similitudo scilicet perfecta, genita et expressa ab intellectu; et hoc totum expressum est verbum, et est totum rei dictae expresivum, et totum in quo res exprimitur; et hoc est intellectum principale quia res non intelligitur nisi in eo. Verbum igitur cordis est ultimum. Est enim tamquam speculum in quo res cernitur, sed non excedens id quod in eo cernitur. Efficitur enim opere naturae ut in eo aliquid cernatur; natura autem non agit aliquid superflue, et ideo non excedit speculum hoc id quod in eo cernitur». De Natura Verbi Intellectus, Opuscula Philosophica, ed. Marietti 1954, n.275, p.94. Este opúsculo se lo considera auténtico
por la mayoría de los críticos a excepción de Mandonet.

5 «Intellectus habet apud se similitudinem rei intellectae, secundum quod rationes incomplexorum concipit: non tamen propter hoc ipsam similitudinem diiudicat, sed solum cum componit vel dividit. Cum enim intellectus concipit hoc quod est animal rationale mortale, apud se similitudinem hominis habet; sed non propter hoc cognoscit se hanc similitudinem habere, quia non iudicat hominem esse animal rationale mortale; et ideo in intellectus habet similitudinem rei intellectae, sed super ipsam similitudinem reflectitur, cognoscendo diuiudicando ipsa. In VI Metaphisicorum», lect. 4, n.1236, ed. Marietti.

6 «Idem non adaequatur sibi ipsi, sed aequalitas diversorum est. De Veritate», q.1, a.3. 
requiere siempre dos términos, de lo contrario no tendría sentido seguir llamándola adecuación), lo que sólo es posible si el concepto es un preconocido como imagen. En realidad, conocer el concepto como imagen es eo ipso conocer la cosa que representa el concepto como imagen. El concepto permite conocer lo otro que sí sin salir de sí mismo, esto es, que el conocimiento es una operación por antonomasia inmanente.

Una nota respecto de lo anterior parece conveniente. La inmanencia de la operación cognoscitiva implica además la veritas ontologica causalis, que es la aptitud que todo ente tiene precisivamente considerado para poder ser conocido por el entendimiento. En este sentido, la verdad ontológica no es en cuanto tal patencia alguna, ni des-ocultación (Unverborgenheit), como algunos comentaristas de cierta influencia heideggeriana han querido ver, sino que es mera posibilidad radical (capacidad, aptitud) de manifestarse, de ser conocido. La teoría de la adecuación supone que ser-inteligible no es lo mismo que estarsiendo-conocido por el intelecto humano. La expresión «ens et verum convertuntur» significa que el ente precisivamente considerado es verdadero, esto es, apto para ser conocido sin error. El ente es radicalmente manifestable al intelecto, pero no manifiesto in actu. ${ }^{7} \mathrm{La}$ absoluta manifestabilidad actual sólo se daría en el pensamiento del Aquinate ante una mente infinita, no por su mero ser intencional (de lo contrario los meros entes de razón tendrían que existir), sino por la absoluta infinitud de su intelecto.

Lo que el intelecto hace es hacer manifiesto el ente. Pero lo entendido en primer lugar y esencialmente por el intelecto es lo que el intelecto concibe de si mismo de la cosa entendida: ${ }^{8}$ el concepto como imagen es lo que (quod) se conoce, es decir, que por ser imagen es esencialmente relativo a la cosa que representa, reenvía a ella a quien la considera. La cosa entendida-res intellecta- es objeto de intellección a través del concepto que la representa, ${ }^{9} \mathrm{de}$ tal suerte, que lo que se comporta de un modo representativo no es, estricta y formalmente, el propio acto de entender (la intelección), sino el concepto. Pues bien, para que el concepto se pueda comportar así, se requiere a su vez que él sea también objeto de intelección, y serlo además de una manera inmediata. Incluso las propias palabras que significan las cosas no lo hacen inmediatamente, sino a través del intelecto, no en cuanto potencia intelectiva, ni como acto inmanente, ni como especie impresa, sino como concepto, por esto mismo, al concepto también se lo llama verbo interior. ${ }^{10} \mathrm{El}$ intelecto conoce el concepto pero en su mediación

7 El ente tiene la aptitud de ser representable, él no es una actividad inteligible, sino sólo en potencia. Ahora bien, el concepto de lo pasivamente representable por la potencia cognoscitiva presupone necesariamente el concepto recíproco de una potencia capaz de representarlo activamente.

8 «Hoc est ergo primo et per se intellectum quod intellectus in seipso concipit de re intellecta (...). Hoc autem sic ab intellectu conceptum dicitur verbum interius, hoc enim est quod significatur per vocem. Non enim vox exterior significat ipsum intellectum (la potencia intelectiva), aut formam ipsius intelligibilem (la especie impresa), aut ipsum intelligere (la operación intelectiva), sed conceptum intellectus, quo mediante significat rem; ut cum dico homo, vel: homo es animal». De Potentia, q.9, a.5.

9 Aquí el término «representación» está tomado en su sentido vicarial y no en su sentido lato que antes se indicó (ver nota n.1).

10 Dico autem intentionem intellectam id quod intellectus in seipso concipit de re intellecta. Quae quidem in nobis neque est ipsa res quae intelligitur, neque est ipsa substantia intellectus; sed est quaedam similitudo 
intencional que refiere al objeto, por esto, el intelecto no conoce el concepto en su ser subjetivo (como afección del intelecto, esto es propio de la reflexión judicativa), sino en su ser objetivo. Es decir, el intelecto no conoce el concepto como cosa sino como imagen. ${ }^{.1}$

El intelecto tiene dos términos, uno físico y otro intencional. El término físico es el propio concepto, que se distingue realmente ${ }^{12}$ del propio acto de intelección. ${ }^{13}$ Este detalle es importante, porque manifiesta que el mismo intelecto es algo expresado por el propio concepto, es decir, que un concepto significa su objeto adecuado y expresa la esencia del intelecto, lo que apoya aún más la tesis de que el concepto tiene que ser lo que es antes conocido, en este aspecto como término de su acto de saberse a sí mismo. ${ }^{14}$ El término intencional cabe considerarlo de dos formas, como término intencional inmediato, entonces corresponde al concepto, y como término intencional mediato, que corresponde a la cosa misma. Esto puede deducirse de dos importantes textos de Tomás de Aquino. El primero muestra como al entender se forma el concepto como intención entendida: «Por el hecho de que la especie inteligible [especie impresa], que es la forma del entendimiento y el principio del entender, es como una semejanza [la especie impresa] de la cosa exterior, se sigue que el .entendimiento forma una intención [al entender se forma el concepto] semejante a dicha cosa, pues cada cual obra con arreglo a lo que es. Y por el hecho de que la intención entendida [el

concepta in intellectu de re intellecta, quam voces exteriores significant; unde et ipsa intentio, verbum interius nominatur; quod est exteriori verbo significatum. Et quidem quod praedicta intentio non sit in nobis res intelecta, inde apparet quod aliud est intelligere rem et aliud est intelligere ipsam intentionem intellectam, quod intellectus facit dum super suum opus reflectitur; unde et aliae sientiae sunt de rebus, et aliae de intentionibus intellectis. IV Contra Gentiles, c.11, n.3466, Ed. Marietti 1961.

11 «Et quidem praedicta intentio non sit in nobis res intellecta, inde apparet quod aliud est intelligere rem, et aliud est intelligere ipsam intentionem intellectam; quod intellectum facit dum super suum opus reflectitur». IV Contra Gent., c.11, n.3466. No se debe ver este texto como una recusación del concepto como lo preconocido, puesto que en esta cita Tomás de Aquino no describe el concepto como imagen, sino como accidente o afección de intelecto. Es el juicio el que puede objetivar el concepto como accidente y su propio acto.

12 «Verbum Dei est ipsum esse divinum et essentia eius, et ipse verus Deus (...); oportet quod in homine intelligente seipsum, verbum interius conceptum non sit homo verus, naturale hominis esse habens; sed sit homo intellectus tantum, quasi quaedam similitudo hominis veri ab intellectu apprehensa (...). Verbum enim hominis non posset dici simpliciter et absolute homo, sed secundum quid, scilicet homo intellectus; unde haec falsa esset homo est verbum; sed haec vera potest esse: homo intellectus est verbum». Contra Gentiles, $1, \mathrm{IV}, \mathrm{c} .11, \mathrm{n} .3471$.

13 El entender es una acción inmanente u operación, en donde el agente es la facultad de entender, la operación o acción inmanente es el entender y el resultado o efecto real interior es el concepto: «sicut in actibus exterioribus est considerare operationem et operatum, puta aedificationem et aedificatum; ita in operibus rationis est considerare ipsum actum rationis, qui est intelligere et ratiotinari, et aliquid per huiusmodi actum constitutum. Quod quidem in speculativa ratione primo quidem est definitio; secundo, enunciatio; tertio vero, syllogismus vel argumentatio». Summa Theologicae, I-II, q.90, a.1 ad 2.

El concepto como producto del intelecto que permanece en él, manifiesta, además de que el acto es inmanente, que el concepto es un accidente del intelecto. «Ipsa enim conceptio est effectus actus intelligendi; unde etiam quando mens intelligit seipsam, eius conceptio non est ipsa mens sed aliquid expresum a notitia mentis». De Veritate, q.4, a.2.

14 Se ha de notar, que la producción del concepto por el acto de entender no es una mera producción, sino que porta una índole importante, es una producción manifestativa de lo aprehendido, es apofántica. De esta suerte, el acto de enteder es una dictio, una locutio interna, en cuanto comporta algo dicho interiormente, el concepto
o verbo interior. 
concepto] es semejante a la cosa, si sigue que el entendimiento, formando dicha intención, entiende la cosa». ${ }^{15}$ Del segundo texto se puede extraer la tesis de los dos términos intencionales subordinados, el inmediato (el concepto) y el mediato (la cosa extramental): «El verbo (el concepto) de nuestro entendimiento es aquello que constituye el término de la operación de nuestro entendimiento, es decir, lo entendido, que se llama también concepción del entendimiento (el concepto), ya se trate de una concepción que se expresa por una voz simple, como sucede cuando el entendimiento forma las quiddidades de las cosas, ya por una voz compleja, lo que ocurre cuando el entendimiento compone o divide». ${ }^{16} \mathrm{El}$ concepto es, por consiguiente, el término interior e inmediato que intercede en la intención del término exterior, la cosa representada. ${ }^{17}$ En este sentido, el concepto es lo que se entiende en el conocimiento intelectual. Se puede dar la siguiente síntesis de lo que se ha dicho: el intelecto entendiendo concibe y forma una intención, que es la índole de la cosa, el concepto o palabra interior. ${ }^{18}$

El Aquinate a veces afirma que lo «primeramente y por sí entendido» (primo et per se intellectum) es el concepto, pero esto no indica que el concepto sea entendido directamente e indirectamente la cosa, como si el concepto tuviera que ser objeto antes de serlo la cosa por él representada. Un conocimiento directo del concepto sólo sería posible en el conocimiento humano mediante una reflexión objetivante. Por tanto, aunque el concepto sea lo inmediatamente conocido, no lo es de un modo directo, sino indirecto -como intentaré mostrar más adelante-. Luego, la expresión «primo et per se» se lo ha de entender en el sentido de «inmediato» y no «directo». El siguiente texto puede esclarecer algo: «Lo que es inmediatamente (per se) entendido no es la cosa de la que se tiene noticia por el entendimiento, puesto que dicha cosa, muchas veces sólo es entendida en potencia y está fuera del que entiende, como cuando el hombre entiende cosas materiales, por ejemplo, una piedra o un animal o algo

15 Contra Gent., 1.I, c.53. «[El concepto o noticia] dupliciter potest considerari. Vel secundum quod comparatur ad cognoscentem: et sic inest cognoscenti sicut accidens in subiecto, et sic non excedit subiectum, quia nunquam invenitur inesse alicui nisi menti. Vel secundum quod comparatur ad cognoscibile: et ex hac parte non habet quod insit, sed quod ad aliud sit. Illud autem quod ad aliquid dicitur, non habet rationem accidentis ex hoc quod est ad aliquid, sed solum ex hoc quod inest; et inde est quod sola relatio secundum rationem sui generis cum substantia remanet in divinis, nec tamen est ibi accidens; et propter hoc notia secundum considerationem istam non est in anima sicut in subiecto, et secundum hanc comparationem excedit mentem in quantum alia a mente per notiam cognoscantur». Quodlibetales VIII, q.1, a.4 c.

16 De Veritate, q.4, a.2 c.

17 «Est autem de ratione interioris verbi, quod est intentio intellecta, quod procedat ab intelligente secundum suum intelligere, cum sit quasi terminus intellectualis operationis; intellectus enim intelligendo concipit et format intentionem sive rationem intellectam, quae est interius verbum». Contra Gentlies, 1.IV, c.11, n.3473. En estos textos se advierte la identificación entre intentio intellecta, verbum mentis, similitudo concepta, etc.

18 El concepto puede ser incomplejo, cuando forma quididades de las cosas, pero también puede ser complejo cuando proviene de la operación del juicio, como aquello en lo que descansa. Pero en ambos casos, el concepto no puede escapar a la conciencia, esto es, debe ser lo que se conoce de modo inmediato. «Verbum intellectus nostri est id ad quod operatio intellectus nostri terminatur, quod est ipsum intellectum, quod dicitur conceptio intellectus; sive sit conceptio significabilis per vocem incomplexam, ut accidit quando intellectus format quidditates rerum; sive per vocem complexam, quod accidit quando intellectus componit et dividit». De Veritate, q.4, a.2. 
parecido; sin embargo, es necesario que lo entendido esté en el entendimiento y sea uno con él. Tampoco lo inmediatamente (per se) entendido es la semejanza de la cosa entendida (la especie impresa), por la cual el entendimiento es informado para entender. Desde luego, el entendimiento no puede entender sino en tanto que está en acto por medio de esa semejanza (especie impresa), como nada puede obrar en tanto que está en potencia, sino en tanto que está en acto por alguna forma. Por tanto, esa semejanza se comporta en el entendimiento como principio del entender, lo mismo que el calor es el principio del calentar, y no como el término del entender. Pero lo que es primeramente e inmediatamente entendido es lo que el entendimiento concibe en sí mismo acerca de la cosa entendida (primo et per se intellectum, quod intellectus in seipso concipit de re intellecta)». ${ }^{19}$ Lo mismo se tendrá que decir de los conceptos compuestos formados por la operación del juicio que divide o compone dos conceptos simples: «El asentir no significa un movimiento del entendimiento a la cosa, sino más bien a la concepción de la cosa, que se tiene en la mente, y a la cual asiente el entendimiento en tanto
que juzga que ella es verdadera». ${ }^{20}$

La cosa exterior «existe en» (inexiste) en el concepto como su semejanza, en este sentido, el concepto puede calificárselo de dos maneras: $1^{\circ}$ ) como índole ejemplar si se lo refiere como principio, $2^{9}$ ) como índole de imagen, si se refiere a lo asemejado como a su principio. ${ }^{21}$ Esto manifiesta que la intelección no permanezca en el propio concepto, sino que su sentido de imagen reenvía a la cosa. La semejanza por sí misma no reenvía a la cosa, para ello se requiere que sea conocida en su relación al origen, y este referir intencional de la semejanza al ser conocida, es lo que se llama imagen. Por eso, el concepto como imagen requiere el ser antes conocido: la imagen para conducir la intención a la cosa debe ella antes dejarse intencionar.

Una de las notas de la índole del concepto que lo caracteriza como concepción, es ser lo entendido en acto internamente. ${ }^{22}$ Para esta función, no difiere esencialmente si es inteligido por semejanza de la cosa extramental -como es el caso de nuestro conocimiento-, o por esencia -es el caso de la esencia divina, de la que todos los seres son sus semejanzas por participar ontológicamente de su ser-. Lo propio de esta nota del concepto es ser la palabra interior (verbum mentis), 10 entendido internamente..$^{23}$ Ahora bien, esto acentúa que el concepto es quod intelligitur, que hay que completar con la otra nota, a saber, que es también un medio en el que se entiende otro que sí mismo.

19 De Potentia, q.9, a.5 c.

20 De Malo, q.6, a. único, ad 14.

21 «Verbum autem interius conceptum est quaedam ratio et similitudo rei intellectae. Similitudo autem alicuius in altero existens, vel habet rationem exemplaris si se habeat ut principium; vel habet potius rationem

22 «Verbum interius estips similitudo sicut ad principium». Contra Gentiles, 1.IV, c.11, n.3474. per intellectum». De Veritate, q.4, a.1.

23 "Quantum ad rationem verbi pertinet, non differt utrum aliquid intelligatur per similitudinem vel essentiam. Constat enim quod interius verbum significat omne illud quod intelligi potest, sive per essentiam sive per similitudinem intelligatur; et ideo omne intellectum, sive per similitudinem sive per essentiam intelligatur,
potest verbum interius dici». De Veritate 
El concepto no es sólo preconocido al objeto extramental, sino que es también una cierta unidad con éste. Esta unidad es quidditativa, a saber, que la índole extramental (que se da subjetivamente) es una con la índole representada en el concepto (que se da objetivamente). Es una identidad específica, no individual, ya que la índole representada en el concepto como algo individual es radicalmente distinta de la dada subjetivamente en la cosa extramental . Esta unidad especifica radica en su ser semejante: ${ }^{24}$ el modo de existir es distinto, pero la manifestación tiene unidad de semejanza.

No puede haber perfecta identidad, entre otras razones, por las siguientes dos: $\left.1^{\mathfrak{a}}\right)$ porque el concepto se relaciona con el objeto extramental como su forma vicaria y no como su forma propia, y $2^{\mathrm{a}}$ ) porque el concepto como signo formal implica alteridad entre el signo y lo representado, de lo contrario se eliminaría de la noción del concepto la nota de ser signo (formal). Es esencial al concepto de signo la alteridad.

La unidad que hay entre el concepto y la cosa que representa no implica que toda la forma de la cosa se poseída por el concepto, ya que en todo conocimiento por semejanza, su modo de conocer es según la conveniencia de esa semejanza con la cosa, que no es según su ser físico. La semejanza puede representar la cosa de tres formas: $1^{a}$ ) por modo de especie, que es el modo más perfecto de representarla, $2^{\mathrm{a}}$ ) por modo de género, ya que la semejanza no alcanza la formas diferenciales de la forma de la cosa, y $3^{\mathfrak{a}}$ ) por modo de analogía, cuando ni siquiera se puede tener una univocidad genérica, este sería el caso de conocer la sustancia real por medio de la semejanza representada de un accidente. Esto indica que el grado de perfección del conocimiento depende del modo como el concepto represente la cosa. ${ }^{25}$ Esto es posible porque la unidad concepto y cosa no es física sino por semejanza, y por tanto no es una unidad absoluta, sino una cierta unidad.

La unidad por semejanza permite decir, junto con los anteriores hallazgos, que la intelección termina en la cosa en el concepto, que es el concepto de la cosa: es el mismo movimiento de intelección el que va al concepto como imagen que el que va a la cosa extramental. ${ }^{26}$ Precisamente por ser el concepto un medio -como imagen-para conocer la cosa es antes preconocido, es el primum cognitum por el intelecto, pues el concepto es lo que hace que la cosa sea en acto inteligible. Para el intelecto expresarse en el concepto es inteligibilidad

24 «Verbum nostri intellectus, ex ipsa $r e$ intellecta habet ut intelligibiliter eamdem naturam contineat».IV Contra Gentiles, c.14, n.3500. «Species intelligibilis est similitudo ipsius essentiae rei, et est quodammado ipsa quidditas et natura rei secundum esse intelligibile, non secundum esse naturale, prout est in rebus». Quodlib., a.3, ad 2. «Species intelligibilis est similitudo ipsius essentiae rei, et est quodammodo ipsa quidditas et natura rei secundum esse intelligibile, non secundum esse naturale, prout est in rebus». Quodlib., a.4.

25 In omni cognitione quae est per similitudinem, modus cognitionis est secundum convenientiam similitudinis ad illud cuius est similitudo; et dico convenientiam secundum representationem, sicut species in anima convenit cum re quae est extra anima: non secundum esse naturale. Et ideo, si similitudo deficiat a repraesentatione speciei, non autem a representatione generis, cognoscetur res illa secundum rationem generis, non secundum rationem speciei. Si autem deficiat etiam a representatione generis, representaret autem secundum convenientiam analogiae tantum: tunc nec etiam secundum rationem generis cognoscretur, sicut si cognosceretur substantia per similitudinem accidentis. De Veritate, q.8, a.1.

26 Cfr. S. Thomae, De Memoria et Reminiscentia, c.1, lect.3. 
en acto de la cosa, sin el cual no hay intelección, de donde, el concepto como imagen es por naturaleza lo anterior. Por eso dice Tomás de Aquino, siguiendo al Estagirita: «Eadem operatione intelligo intelligibile et intelligo me intelligere», ${ }^{27}$ en donde «entender que entiende» debe incluir no sólo al acto sino también al concepto expresado. Aplicando analógicamente la teoría hilemórfica (que en sentido estricto no se puede aplicar) se puede decir, que como la razón de conocer es la semejanza conceptual de la cosa (la forma de la cosa en cuanto conocida), entonces la razón de conocer -que se comporta como forma del conocimiento-forma una unidad intencional con la cosa-que se comporta como la materia del conocimiento-, paralelamente a como la forma física y la materia constituyen una unidad en, el ser..$^{28}$

\section{EL CONCEPTO ES ESENCIALMENTE ALTERIDAD}

Habida cuenta de todos estos desarrollos, parece que la inmanencia del conocimiento dificulta la tesis del realismo. Esto sería verdad si se concibe los conceptos como meras afecciones del intelecto. Pero precisamente esto era lo que había que evitar. La afirmación del realismo tiene sentido cuando los conceptos son conocidos como efectivamente representativos, esto es, conocer el concepto como lo que es imagen. La noción de imagen juega, por tanto, un papel capital en el juicio que afirma que el intelecto alcanza en un sólo movimiento las cosas. El concepto como imagen no es $\sin$ la cosa, hay una unidad entre ambos, en donde el concepto está reificado, porque es imagen de la cosa: el ser de la imagen es precisamente un puro reenviar a su término relativo: «La concepción del entendimiento se ordena a la cosa entendida como a su fin, pues el entendimiento forma en sí la concepción de la cosa para conocer la cosa entendida ${ }_{,}^{29}$ en este sentido, el concepto como término intencional inmediato, o interno, es calificado a veces por el Aquinate como un «cuasi término» de la operación intelectual. ${ }^{30} \mathrm{La}$ afirmación del realismo pierde su sentido cuando no se toma el concepto objetivamente tomado, en su ser imagen.

Este modo de hablar, en el que se dice de un objeto está «fuera» o «dentro» del .pensamiento ha inducido a decir a J. Maritain que «argumentar con el sentido espacial y material metafóricamente evocado por ese «dentro» y por el «fuera» que le corresponde es el

27 I Senten., d.1, q.2, a.1, ad 2. Otros textos: «Non alio actu potentia fertur in obiectum et in actum suum; eodem enim actu intellectus intelligit se et intelligit se intelligere».I Sente., d.10, q.1, a.5, ad 2.

28 «Illud in quo aliquid videtur est ratio cognoscendi illud quod in eo videtur. Ratio autem cognoscendi est forma rei in quantum est cognita, quia per eam fit cognitio in actu; unde sicut ex materia et forma est unum esse, ita ratio cognoscendi et res cognita sunt unum cognitum, et propter hoc utriusque, in quantum hiusmodi, est una cognitio secundum habitum et secundum actum; et ita non est alius habitus (nec alius actus) quo cognoscitur verbum et ea quae in verbo videntur». III Sent., d.14, q.1, 4 in sol. También: «Intellectus humanus qui fit in actu per speciem rei intellectae, per eandem speciem intelligitur sicut per formam suam». Summa The., I, q.87, a.1, ad 3.

29 De Potentia, q.8, a.1 c.

30 Cfr. Contra Gentiles, lib.I, c.53, n.444. 
sofisma trivial del idealismo». ${ }^{31}$ Maritain parece exagerar algo el sentido de la metáfora espacializante, especialmente en su equiparación al inmanentismo radical. El pensamiento del Aquinate sería un clausurado inmanentismo si equiparase la necesidad del pensamiento para que algo pueda ser pensado con la necesidad del mismo pensamiento para que algo pueda ser. Por lo demás, la metáfora espacial me parece legítima y útil para mostrar la inmanencia del acto de conocer en la expresión «el ser de lo conocido en el cognoscente». Ciertamente, no es más que una metáfora, realmente no se puede hablar de un «dentro» y de un «fuera», sino más bien de que el poder de la inmaterialidad del conocimiento es la absoluta inmaterialidad, en donde no ha lugar para que una cosa sea exterior a otra cosa externa, sino que el conocimiento es una forma de vida superior a lo que es espacial, en la medida en que sin salir de sí puede perfeccionarse con lo que es ajeno a él.

El concepto es alteridad en la medida que es esencialmente imagen, relativo a, que Tomás de Aquino llama «id in quo». Decir que la imagen lo es de sí mismo es una contradictio in adiectis, puesto que la imagen lo es siempre de otro que sí, alteridad. Alteridad indica aquí no la mera relación del concepto al objeto, sino la esencial respectividad o referencia a él. El concepto no es sólo lo que se ve, sino también en lo que algo otro se ve, es decir, es el midio en el que (medium in quo) el entendimiento aprehende su objeto directo. El propio concepto, en su ser subjetivo se oculta al movimiento directo del intelecto que conoce la cosa extramental, es como un silencioso intermediario entre entendimiento y cosa entendida, su intelección (el concepto como quod intelligitur) hace que por él (in quo) se alcanze la cosa, de tal suerte, que lo entendido es tanto el propio concepto como la cosa. ${ }^{32}$

El concepto es lo pre-conocido en la intelección de la cosa, luego necesariamente debe haber un orden de prioridades. Lo primero entendido es el concepto, lo segundo la cosa entendida. Este orden de prioridad no es temporal, ya que el concepto no es el término intencional directo, sólo inmediato, es decir, que en su función mediadora no se deja notar. No sucede que primero se conozca el concepto y después la cosa, sino que la prioridad del conocimiento del concepto es únicamente de naturaleza, aunque temporalmente ambos son simultáneos. La prioridad temporal implicaría problemas indeseables como el de la doble aprehensión, mientras que la concepción es un solo movimiento el que entiende el concepto y el que entiende la cosa. ${ }^{33}$

31 Maritain, J., Oeuvres complètes IV, 418.

32 «Conceptio intellectus est media inter intellectum et rem intellectam, quia ea mediante operatio intellectus pertingit ad rem; et ideo conceptio intellectus non solum est $i d$ quod intellectum est, sed etiam in quo res intelligitur; ut sic quod intelligitur possit dici et res ipsa et conceptio intellectus». De Veritate, q.4, a.2, ad 3.

33 «Duplex est motus animae in imaginem: unus quidem in imaginem ipsam secundum quod est res quaedam; alio modo, in imaginem inquantum est imago alterius. Et inter hos motus est haec differentia, quia primus motus, quo quis movetur in imaginem prout est res quaedam, est alius a motu qui est in rem: secundus autem motus, qui es in imaginem inquantum est imago, est unus et idem cum illo qui est in rem». Summa Theologiae, III, q.25, a.3 c. Cfr. también De Veritate, q.22, a.14 c. 
El concepto como aquello en lo que otro se ve, es su razón de conocimiento, que es la forma de la cosa en cuanto conocida. ${ }^{34}$ Algo da a conocer a otro de dos formas: $1^{\mathrm{a}}$ ) como aquello de cuyo conocimiento se deriva el conocimiento de otra cosa, como se conocen las conclusiones por las premisas, pero esto no es el que se da en el concepto, y $2^{2}$ ) como aquello en lo cual se conoce otra que sí mismo. ${ }^{35}$ Por lo tanto, el concepto no es un por lo cual se ve la cosa, sino un en el cual se la ve.

El concepto es lo que se conoce y es alteridad. Esta doble propiedad del concepto le viene precisamente por ser imagen: la imagen es mostración de sí misma, y mostrándose muestra a otro distinto de sí. Lo que es la imagen es inseparable de su ser alteridad. El intelecto no puede salir de sí mismo, todo lo entiende dentro de sí, pero como un «en lo que» se entiende la cosa extramental.

El concepto no es alteridad por ser simple semejanza, sino por ser imagen. También la especie impresa es semejanza de la cosa de la que proviene, sin embargo, ella no es esencialmente alteridad, no remite por sí misma a la cosa de la que es origen. Además, no es conocida directamente en el movimiento intelectivo, sino algo por lo cual entendemos, sin llegar a ser algo en lo cual $^{36} \mathrm{La}$ especie impresa es principio de intelección pero no lo que se conoce y en lo cual se conoce la naturaleza de las cosas ${ }^{37}$ El concepto es semejanza e imagen. Es semejanza por ser lo expresado por el intelecto a partir de la especie impresa y es imagen además por ser término inmediato del conocimiento que reenvía a la cosa. Si conociéramos directamente la semejanza de todos nuestros conocimientos, éstos serían verdaderos. ${ }^{38} \mathrm{La}$ imagen añade a la semejanza su estar-siendo-conocida, no en su ser subjetivo, sino objetivo.

34 «Illud in quo aliquid videtur, est ratio cognoscendi illud quod in eo videtur. Ratio autem cognoscendi est forma rei in quantum est cognita, quia per eam fit cognitio in actu; unde (...) ratio cognoscendi et res cognita sunt unum cognitum; et propter hoc utriusque, in quantum huiusmodi, est una cognitio secundum habitum et secundum actum; et ita non est alius habitus (nec alius actus) quo cognoscitur verbum et ea quae in verbo videtur». III Sent., d.14, q.1, a.1, in sol.

35 «Dupliciter aliquid aliquo dicitur cognoscendi: uno modo sicut ex cuius cognitione deveniatur in cognitionem ipsius, et sic dicuntur conclusiones principiis cognosci; et hoc modo non potest aliquis cognosci seipso. Alio modo dicitur aliquid cognosci sicut in quo cognoscitur, et sic oportet ut id quo cognoscitur alia cognitione cognoscitur 'quam id quo cognsocitur eo». De Veritate, q.10, a.8, ad 9.

36 «Istud ergo sic expresum, scilicet formatum in anima, dicitur verbum interius; et ideo comparatur ad intellectum non sicut quo intelligit, sed sicut in quo intelligit; quia in ipso expreso et formato videt naturam rei intellectae». Comment. Evan. S. Ioannis, c.I, v.1; ed. Marietti 1952, n.25, p.8.

37 La especie inteligible impresa es la forma por la cual el entendimiento entiende (cfr. Summa Theologicae, I, q.85, a.2 c.), de tal suerte, que el entendimiento informado por la especie impresa, forma en sí mismo, al entender, el concepto, una semejanza que es también imagen de la cosa entendida (cfr. Contra Gentiles, lib.I, c.53, n.443). Por tanto, la especie impresa es la forma del intelecto, mientras que el concepto es lo formado por él.

38 «[Las especies impresas] se habent sicut id quo intelligimus: 1) Quia similitudo rei intellectae quae est species intelligibilis, est forma secundum quam intellectus intelligit rem; sicut similitudo rei visibilis in oculto est secundum quam visus videt rem ipsam. (...) [Además, si fueran conocidas en sí mismas] quod intelligitur esset verum. Tamen, quia intellectus supra seipsum reflectitur, secundum eamdem reflexionem intelligit et suum intelligere, et speciem qua intelligit; et sic species intellecta est id quod secundario intelligitur; primo autem quod intelligit est res ipsa, cuius species intelligibilis est similitudo». Summa Th., I, q.85, a.2. El término «especies inteligibles» se refiere a las especies impresas. 
La especie inteligible impresa es una realidad psíquica ${ }^{39}$ que es además semejanza de otra cosa, pero de otra forma que la imagen conceptual. En este sentido se expresa Tomás de Aquino: «Aunque ambos, a saber, la especie (impresa inteligible) y el verbo (la imagen conceptual) engendrado por ella sean un accidente, sin embargo, el verbo (la imagen conceptual) es más semejanza de la sustancia que la especie (impresa) $\gg .^{40} \mathrm{La}$ semejanza que porta la especie impresa es causada por la cosa extramental, mientras la de la imagen es además una relación intencional al objeto.

39 La especie impresa se la incluye dentro de la categoría del accidente cualidad, y dentro del género de la cualidad, es de la especie hábito en sentido estricto, es decir, una disposición permanente.

40 De Natura Verbi Intellectus, c.2, n.281. Se ha de notar, que el momento de la especie impresa, aunque necesaria para el conocimiento, no es un momento cognscitivo, sino precognoscitivo: «Ser movido por el objeto no pertenece a la razón del cognoscente en tanto que cognoscente, sino en tanto que potencia cognoscente». Summa Theologica, I, q.56, a.1 c. La función de la especie impresa es la de estímulo para actualizar el conocimiento. 